\title{
Advances in clinical research in chronic kidney disease
}

\author{
Yutong Zou' ${ }^{1}$, Fang Liu', Mark E. Cooper ${ }^{2}$, Zhonglin Chai ${ }^{2}$ \\ 'Division of Nephrology, West China Hospital of Sichuan University, Chengdu 610041, Sichuan Province, China; \\ ${ }^{2}$ Department of Diabetes, Central Clinical School, Monash University, Melbourne 3800, Australia
}

\section{INTRODUCTION}

Current international guidelines define chronic kidney disease (CKD) as an abnormality of kidney function or structure that is present for at least 3 months, regardless of underlying causes, with implications for health. ${ }^{[1]}$ The prevalence of CKD varies worldwide due to differences in socioeconomic conditions and ethnicity. Indeed, about 1 in 10 people have CKD in high- and middle-income countries, and this is even higher in low-income countries. CKD occurs primarily as a result of diabetes, hypertension, obesity, or glomerulonephritis. ${ }^{[2,3]}$ Although current treatment strategies can improve the renal function to some extent, patients are still at high risk of progression to end-stage renal disease (ESRD) or allcause death in the long term. Since the prevalence of CKD is an increasing public health issue and poses a significant social as well as medical and economic burden, effective drugs to preserve kidney function are urgently needed.

As recently known, correction of glomerular hyperfiltration, management of metabolic abnormalities, and addressing the risk factors associated with kidney injury, such as diabetes, hypertension, hyperlipidemia, obesity, and smoking, are effective in slowing down the progression of CKD. ${ }^{[4]}$ Furthermore, a number of recent clinical studies have demonstrated promising specific renoprotection conferred by several classes of new agents, which can be used to optimize the management of patients with CKD.

Angiotensin converting enzyme inhibitors (ACEIs) and angiotensin II receptor blockers (ARBs) have been demonstrated as renoprotective agents. These effects are attributed to their actions as antihypertensive and anti-proteinuric agents. ${ }^{[5]}$ Moreover, the agents that interrupt the renin-angiotensin system have also been shown to attenuate renal damage via a range of mechanisms including reversal of chronic tubulointerstitial hypoxia, reducing the interstitial infiltration of inflammatory cells, decreasing iron deposition, inhibiting intrarenal activity of the plasminogen activator inhibitor (PAI-1), and acting as antifibrotic agents by inhibiting prosclerotic growth factors such as transforming growth factor beta (TGF- $\beta$ ) in various forms of $\mathrm{CKD}^{\left[{ }^{[6,7]}\right.}$ Despite the widespread use of ACEI/ARB, patients with CKD still have a high residual risk of progressing to ESRD.

To further interrupt the reninangiotensin-aldosterone system (RAAS), mineralocorticoid receptor antagonists (MRAs), which block the action of aldosterone, can reduce fibrosis in many target organs, including blood vessels, kidneys, and heart. ${ }^{[8]}$ The recently developed new generation of nonsteroidal MRAs such as finerenone appear to have less side effects such as hyperkalemia, thus making these drugs suitable for use in patients with CKD. ${ }^{[9]}$ Phase III randomized clinical trials confirmed that finerenone therapy resulted in a lower risk of CKD progression and cardiovascular morbidity than placebo in patients with type 2 diabetes (T2D) and $\mathrm{CKD} \cdot{ }^{[10]}$ Confirmation of the positive results of the initial finerenone therapy in reducing kidney failure and disease progression in diabetic kidney disease (FIDELIO-DKD) has been reported, albeit not yet fully published in the finerenone in reducing 
cardiovascular mortality and morbidity in diabetic kidney disease (FIGARO-DKD) study, where less-advanced CKD in T2D subjects was studied. In that study, finerenone reduced a composite of cardiovascular death and events. ${ }^{[1]}$ Renal findings are pending for that study.

Notably, a series of recent studies have confirmed that a recently developed oral antidiabetic class of drugs, the sodium-glucose cotransporter-2 (SGLT2) inhibitors, can retard the decline in renal function beyond their hypoglycemic effect by acting in a hemodynamic manner, including reducing hyperfiltration in patients with diabetic kidney disease (DKD), a form of CKD, which is characterized by glomerular and systemic hemodynamic dysregulation. ${ }^{[12]}$ Moreover, according to the results of various cardiovascular outcome trials (CVOTs), including empagliflozin cardiovascular outcomes (EMPA-RE), canagliflozin cardiovascular assessment study (CANVAS), and dapagliflozin effect on cardiovascular events (DECLARE-TIMI) studies, SGLT2 inhibitors have demonstrated not only cardiovascular, but also renoprotective properties. These agents not only reduce cardiovascular events and all-cause mortality in patients with T2D and CKD, but also reduce proteinuria and improve the prognosis, reducing the development of ESRD. ${ }^{[13,14]}$ Indeed, building on these CVOTs, renally dedicated trials have been completed, including canagliflozin on renal and cardiovascular outcomes in participants with diabetic nephropathy (CREDENCE) and dapagliflozin and prevention of adverse outcomes in CKD (DAPA-CKD), and have demonstrated renoprotection in T2D subjects. ${ }^{[15]}$ Furthermore, in the DAPA-CKD trial, non-diabetic subjects with other forms of CKD were also reported to have renoprotection to a similar degree to that seen in T2D. SGLT2 inhibitors reduce proximal tubule sodium chloride reabsorption and increase the delivery of these ions to the macula densa, thereby modulating tubuloglomerular feedback, leading to an increase in the resistance in the afferent arteriole and a reduction in intraglomerular pressure. ${ }^{[16]}$ Furthermore, SGLT2 inhibitors inhibit fluid reabsorption in the proximal tubule, thus increasing the hydrostatic pressure in the tubule. By contrast, ACE inhibitors and ARBs decrease the resistance in the efferent arteriole and can be administered together with SGLT2 inhibitors, complementarily reducing intraglomerular pressure.

Other relatively new hypoglycemic drugs, such as glucagonlike peptide-1 receptor (GLP-1R) agonists, also have shown renoprotective effects in improving albuminuria and/or retarding glomerular filtration rate (GFR); ${ }^{[17,18]}$ but these effects are not as potent as those seen with SGLT2 inhibitors and the mechanism of action of these agents remains difficult to delineate.
Dipeptidyl peptidase-4 (DPP-4) inhibitors are widely used in the management of T2D. These drugs are often used in subjects with or at risk of CKD. Numerous CVOTs have evaluated these agents and, indeed, modest effects on reducing urinary albumin excretion have been observed. ${ }^{[19]}$ However, whether this relates to the associated glucoselowering effects or represents a specific renoprotective effect of these drugs has not been fully elucidated. One of the DPP-4 inhibitors, linagliptin, can be used safely without any dose change at any level of renal function, including in dialysis patients. This has led to several renally oriented trials such as MARLINA in type 2 diabetic subjects with albuminuria ${ }^{[20]}$ and in a large CVOT known as CARMELINA which included a renal primary endpoint. ${ }^{[21]}$ Modest reductions in albuminuria were observed with linagliptin, but no reduction in any hard renal endpoints, as has been seen with other glucose-lowering agents such as SGLT2 inhibitors, was observed. ${ }^{[21]}$

For the treatment of renal anemia in CKD, hypoxiainducible factor (HIF) prolyl hydroxylase ( $\mathrm{PH}$ ) enzyme inhibitors have received much attention as a new drug class stabilizing the HIF-PH axis, stimulating endogenous erythropoietin production, upregulating the expression of transferrin receptor, increasing iron uptake by proerythrocytes, and promoting erythrocyte maturation. ${ }^{[22]}$ Although a recent clinical trial showed increased levels of hemoglobin with one of these inhibitors, roxadustat, ${ }^{[23]}$ further studies are still needed to investigate the effect of such therapies on long-term cardiovascular and renal outcomes. Interestingly, another agent of this class, enarodustat, showed beneficial effects on cardiovascular complications caused by CKD in a rat model. ${ }^{[2]}$

The use of selective endothelin receptor antagonists is another effective and safe approach to reduce albuminuria and blood pressure. ${ }^{[25]}$ The study of diabetic nephropathy with atrasentan (SONAR) has reported that atrasentan decreased the risk of renal events in participants with T2D and $\mathrm{CKD}$, demonstrating its potential benefits in preserving renal function in diabetic patients who otherwise would have a high risk of ESRD. ${ }^{[26]}$

The kidney can be damaged as a result of aberrant activity of the immune system. Approaches to target immune cells have been explored in the clinical setting using biological agents to treat certain kidney diseases. For example, rituximab, a monoclonal antibody that depletes B cells, is increasingly used "off-label" for certain renal conditions such as in renal transplantation and membranous nephropathy, ${ }^{[2]}$ minimal change disease, focal segmental glomerulosclerosis, ${ }^{[28]}$ and lupus nephritis. ${ }^{[29]}$ Furthermore, using biological agents to target the innate inflammatory response, complement and T-cell activation, as well as to 
block systemic inflammation is also being considered as a new frontier of immunosuppressive therapy for CKD. For example, $\mathrm{C}-\mathrm{C}$ chemokine receptor type 2 inhibition with CCX140-B has been observed to have a renoprotective effect in patients with DKD. ${ }^{[30]}$

Stem cell therapies have been considered to be a promising therapeutic strategy to treat CKD through stimulating kidney regeneration. Indeed, there is growing evidence that stem cells, including induced pluripotent stem cells, ${ }^{[31]}$ mesenchymal stem/stromal cells, ${ }^{[32]}$ and renal stem/ progenitor cells, ${ }^{[33]}$ exert their therapeutic effects on CKD by replacing damaged tissues as well as influencing key paracrine pathways. However, further studies are needed to understand the risks of fibrosis, maldifferentiation, or tumor formation and adverse events of immunosuppression, as well as for further validation of the long-term safety and tolerability of these therapies.

Gene editing is also being explored for the treatment of CKD. In contrast to traditional gene therapy approaches, clustered, regularly interspaced short palindromic repeats (CRISPR) gene editing, a state-of-the-art technology that is not only a promising tool for research, but also has great potential to be used for gene therapy, can precisely edit or repair specific disease-causing mutations. ${ }^{[34]}$ Specifically, CRISPR has been explored as a promising approach to treat immune-mediated kidney diseases such as lupus nephritis or immunoglobulin A nephropathy, where T lymphocyte receptors are engineered to recognize specific antigens. ${ }^{[35]}$ Furthermore, CRISPR-Cas gene editing is being explored to alter key relevant genes in large domestic species in order to produce more suitable donor kidneys for transplantation in humans, thereby alleviating the shortage of donor organs. ${ }^{[35]}$

In summary, $\mathrm{CKD}$ is a major burden in human health globally, with a major treatment gap to efficiently deal with this devastating condition. Optimal management of risk factors such as diabetes and hypertension and the use of certain antidiabetic agents such as SGLT2 inhibitors and GLP-1R agonists as well as agents such as ACEI, ARBs, and, more recently, nonsteroidal MRAs appear to retard the progression of CKD as well as show associated cardiovascular benefits. However, despite all these treatments, CKD continues to progress, and therefore, safe and more efficacious treatment strategies are urgently needed. Fortunately, recent advances in basic and clinical research in this area, such as immunotherapy, newly identified drug targets, gene therapy, and CRISPR technology, have shown great potential to provide additional novel technologies and strategies in the near future to more successfully combat CKD.

\section{Conflict of Interest}

None declared.

\section{REFERENCES}

1. Stevens PE, Levin A. Evaluation and management of chronic kidney disease: synopsis of the kidney disease: improving global outcomes 2012 clinical practice guideline. Ann Intern Med 2013; 158:825-30.

2. Webster AC, Nagler EV, Morton RL, Masson P. Chronic kidney disease. Lancet, 2017; 389:1238-1252.

3. Stanifer JW, Muiru A, Jafar TH, Patel UD. Chronic kidney disease in lowand middle-income countries. Nephrol Dial Transplant 2016;31:868-74.

4. Chen TK, Knicely DH, Grams ME. Chronic kidney disease diagnosis and management: A review. JAMA 2019;322:1294-304.

5. Zhang F, Liu H, Liu D, Liu Y, Li H, Tan X, et al. Effects of RAAS Inhibitors in Patients with Kidney Disease. Curr Hypertens Rep 2017;19:72.

6. Izuhara $\mathrm{Y}$, Nangaku M, Inagi R, Tominaga $\mathrm{N}$, Aizawa T, Kurokawa $\mathrm{K}$, et al. Renoprotective properties of angiotensin receptor blockers beyond blood pressure lowering. J Am Soc Nephrol 2005;16:3631-41.

7. Miyata T, van Ypersele de Strihou C. Renoprotection of angiotensin receptor blockers: beyond blood pressure lowering. Nephrol Dial Transplant 2006;21: 846-9.

8. Tesch GH, Young MJ. Mineralocorticoid receptor signaling as a therapeutic target for renal and cardiac fibrosis. Front Pharmacol 2017; 8:313.

9. Capelli I, Gasperoni L, Ruggeri M, Donati G, Baraldi O, Sorrenti G, et al. New mineralocorticoid receptor antagonists: update on their use in chronic kidney disease and heart failure. J Nephrol 2020; 33:37-48.

10. Bakris GL, Agarwal R, Anker SD, Pitt B, Ruilope LM, Rossing P, et al. Effect of finerenone on chronic kidney disease outcomes in type 2 diabetes. N Engl J Med, 2020;383:2219-29.

11. Bayer's finerenone meets primary endpoint in phase III FIGARO-DKD cardiovascular outcomes study in Patients with chronic kidney disease and type 2 diabetes. Whippany, NJ: Bayer; May 10, 2021. Available at: https://media.bayer.com/baynews/baynews.nsf/id/F6AC5A8D4B0647A AC12586D100282B80?open\&ref=irrefndcd. Accessed on June 2, 2021.

12. Cherney DZ, Perkins BA, Soleymanlou N, Maione M, Lai V, Lee A, et al. Renal hemodynamic effect of sodium-glucose cotransporter 2 inhibition in patients with type 1 diabetes mellitus. Circulation 2014; 129:587-97.

13. Zelniker TA, Wiviott SD, Raz I, Im K, Goodrich EL, Bonaca MP, et al. SGLT2 inhibitors for primary and secondary prevention of cardiovascular and renal outcomes in type 2 diabetes: a systematic review and metaanalysis of cardiovascular outcome trials. Lancet 2019; 393:31-9.

14. Johansen ME, Argyropoulos C. The cardiovascular outcomes, heart failure and kidney disease trials tell that the time to use sodium glucose cotransporter 2 inhibitors is now. Clin Cardiol 2020; 43:1376-87.

15. Heerspink HJL, Stefánsson BV, Correa-Rotter R, Chertow GM, Greene T, Hou FF, et al. Dapagliflozin in patients with chronic kidney disease. N Engl J Med, 2020; 383:1436-46.

16. Wilcox C.S. Antihypertensive and renal mechanisms of SGLT2 (sodiumglucose linked transporter 2) inhibitors. Hypertension 2020; 75:894-901.

17. Gerstein HC, Colhoun HM, Dagenais GR, Diaz R, Lakshmanan M, Pais $\mathrm{P}$, et al. Dulaglutide and cardiovascular outcomes in type 2 diabetes (REWIND): a double-blind, randomised placebo-controlled trial. Lancet 2019; 394:121-30.

18. Mann JFE, Ørsted DD, Brown-Frandsen K, Marso SP, Poulter NR, Rasmussen $\mathrm{S}$, et al. Liraglutide and renal outcomes in type 2 diabetes. N Engl J Med, 2017; 377: 839-48.

19. Mosenzon O, Leibowitz G, Bhatt DL, Cahn A, Hirshberg B, Wei C, et al. Effect of saxagliptin on renal outcomes in the SAVOR-TIMI 53 trial. Diabetes Care 2017; 40: 69-76.

20. Groop PH, Cooper ME, Perkovic V, Hocher B, Kanasaki K, Haneda M, 
et al. Linagliptin and its effects on hyperglycaemia and albuminuria in patients with type 2 diabetes and renal dysfunction: the randomized MARLINA-T2D trial. Diabetes Obes Metab 2017; 19:1610-9.

21. Perkovic V, Toto R, Cooper ME, Mann JFE, Rosenstock J, McGuire DK, et al. Effects of linagliptin on cardiovascular and kidney outcomes in people with normal and reduced kidney function: secondary analysis of the CARMELINA randomized trial. Diabetes Care 2020; 43:1803-12.

22. Gupta N, Wish JB. Wish, Hypoxia-inducible factor prolyl hydroxylase inhibitors: a potential new treatment for anemia in patients with CKD. Am J Kidney Dis 2017; 69: 815-26.

23. Chen N, Hao C, Peng X, Lin H, Yin A, Hao L, et al. Roxadustat for anemia in patients with kidney disease not receiving dialysis. N Engl J Med 2019; 381:1001-10.

24. Uchida L, Tanaka T, Saito H, Sugahara M, Wakashima T, Fukui K, et al. Effects of a prolyl hydroxylase inhibitor on kidney and cardiovascular complications in a rat model of chronic kidney disease. Am J Physiol Renal Physiol 2020;318:F388-401.

25. de Zeeuw D, Coll B, Andress D, Brennan JJ, Tang H, Houser M, et al. The endothelin antagonist atrasentan lowers residual albuminuria in patients with type 2 diabetic nephropathy. J Am Soc Nephrol 2014; 25: 1083-93.

26. Heerspink HJL, Parving HH, Andress DL, Bakris G, Correa-Rotter R, Hou FF, et al. Atrasentan and renal events in patients with type 2 diabetes and chronic kidney disease (SONAR): a double-blind, randomised, placebo-controlled trial. Lancet 2019; 393:1937-47.

27. Fervenza FC, Appel GB, Barbour SJ, Rovin BH, Lafayette RA, Aslam
$\mathrm{N}$, et al. Rituximab or cyclosporine in the treatment of membranous nephropathy. N Engl J Med 2019;381:36-46.

28. Gauckler P, Shin JI, Alberici F, Audard V, Bruchfeld A, Busch M, et al. Rituximab in adult minimal change disease and focal segmental glomerulosclerosis - What is known and what is still unknown? Autoimmun Rev 2020; 19:102671.

29. Cassia M, Alberici F, Gallieni M, Jayne D. Lupus nephritis and B-cell targeting therapy. Expert Rev Clin Immunol 2017; 13: 951-62.

30. de Zeeuw D, Bekker P, Henkel E, Hasslacher C, Gouni-Berthold I, Mehling $\mathrm{H}$, et al. The effect of CCR2 inhibitor CCX140-B on residual albuminuria in patients with type 2 diabetes and nephropathy: a randomised trial. Lancet Diabetes Endocrinol 2015; 3:687-96.

31. Caldas HC, Lojudice FH, Dias C, Fernandes-Charpiot IMM, Baptista MASF, Kawasaki-Oyama RS, et al. Induced pluripotent stem cells reduce progression of experimental chronic kidney disease but develop Wilms' tumors. Stem Cells Int 2017; 2017:7428316.

32. Bian XH, Zhou GY, Wang LN, Ma JF, Fan QL, Liu N, et al. The role of CD44-hyaluronic acid interaction in exogenous mesenchymal stem cells homing to rat remnant kidney. Kidney Blood Press Res 2013; 38:11-20.

33. Ronconi E, Sagrinati C, Angelotti ML, Lazzeri E, Mazzinghi B, Ballerini $\mathrm{L}$, et al. Regeneration of glomerular podocytes by human renal progenitors. J Am Soc Nephrol, 2009; 20: 322-32.

34. Miyagi A, Lu A, Humphreys BD. Gene editing: powerful new tools for nephrology research and therapy. J Am Soc Nephrol 2016;27:2940-7.

35. Cruz NM, Freedman BS. CRISPR gene editing in the kidney. Am J Kidney Dis 2018;71: 874-883.

How to cite this article: Zou Y, Liu F, Cooper ME, Chai Z. Advances in clinical research in chronic kidney disease. J Transl Intern Med 2021; 9: $146-9$. 